Artículo científico

Volumen 30(2):483-495. Mayo-agosto, 2019

e-ISSN 2215-3608, doi:10.15517/am.v30i2.34307

https://revistas.ucr.ac.cr/index.php/agromeso/index

\title{
Estabilidad fenotípica de genotipos de Lolium sp. en el trópico alto de Nariño, Colombia ${ }^{1}$
}

\section{Phenotypic stability of genotypes Lolium sp. in the high tropic of Nariño, Colombia}

\author{
Máryory Maricela Cadena-Guerrero², Mario Augusto García-Dávila ${ }^{3}$,Edwin Castro-Rincón ${ }^{2}$
}

1 Recepción: 27 de agosto, 2018. Aprobación: 13 de diciembre, 2018. Este trabajo formó parte de un proyecto de tesis de maestría llevado a cabo en la Corporación Colombiana de Investigación Agropecuaria (AGROSAVIA), con recursos del Sistema General de Regalías, Colombia.

2 Corporación Colombiana de Investigación Agropecuaria (AGROSAVIA), Centro de Investigación Obonuco, Pasto, Colombia. mmcadena@ agrosavia.co; ecastro@agrosavia.co (http://orcid.org/0000-0001-9841-8242).

3 Universidad Nacional de Colombia, Palmira, Colombia. magarciada@unal.edu.co

\section{Resumen}

Introducción. Las especies del género Lolium representan un componente importante en la alimentación bovina en varias regiones de Colombia, sin embargo, en el trópico alto de Nariño, una de las principales cuencas lecheras del país, existe poco conocimiento sobre el comportamiento de los cultivares que manejan los productores; no existen estudios de estabilidad que permitan conocer cuáles de los cultivares que se ofrecen en el mercado se comportan mejor en una determinada localidad, lo cual afecta la economía de los ganaderos. Objetivo. El objetivo de la presente investigación fue determinar la estabilidad fenotípica del rendimiento del forraje verde (RFV) y de la materia seca (RMS) en raigrás, en la cuenca lechera del trópico alto de Nariño. Materiales y métodos. Entre el 2016 y 2017 , se evaluaron diez genotipos en Pasto, Cumbal y Sapuyes. Los experimentos se establecieron bajo un diseño de bloques completos al azar con cuatro repeticiones. Para el análisis de adaptabilidad y estabilidad de RFV y RMS se utilizaron el modelo propuesto por Eberhart y Russell, y el de análisis de efectos principales aditivos e interacciones multiplicativas (AMMI). Resultados. Los genotipos Tetralite II, Bóxer, Bestfor Plus y Aubade presentaron los mayores rendimientos de forraje verde (7,52-8,34 t.ha.corte $\left.{ }^{-1}\right)$ y de materia seca $\left(1,29-1,37\right.$ t.ha.corte $\left.{ }^{-1}\right)$ en los tres ambientes estudiados. Con el modelo Eberhart y Russell, para RFV, estos genotipos fueron clasificados como de mejor respuesta en ambientes favorables y predecibles, y por RMS también fueron predecibles, pero Aubade y Bestfor Plus se clasificaron con buena respuesta en todos los ambientes y Bóxer y Tetralite II, con mejor respuesta solo en ambientes favorables. Conclusión. El modelo AMMI permitió identificar al municipio Pasto como el ambiente más favorable y a los genotipos Bóxer y Tetralite II como los de mejor comportamiento en este ambiente.

Palabras clave: raigrás, adaptabilidad, AMMI, forraje verde.

\begin{abstract}
Introduction. The species of the Lolium genus represent an important component in the bovine feeding in several regions of Colombia, however, in the high tropic of Nariño, one of the main dairy basins of the country, there is little knowledge about the performance of the cultivars managed by the producers; there are no stability studies that allow us to know which of the cultivars offered in the market behave better in a specific location, which affects the
\end{abstract}


farmers' economy. Objective. The objective of this research was to determine the phenotypic stability of the yield of green forage (RFV) and dry matter (RMS) in ryegrass, in the dairy basin of the high tropic of Nariño. Materials and methods. Between 2016 and 2017, ten ryegrass genotypes were evaluated in Pasto, Cumbal, and Sapuyes. The experiments were established under a randomized complete block design with four repetitions. For the analysis of adaptability and stability of RFV and RMS, the model proposed by Eberhart and Russell, and the analysis of principal additives effects and multiplicative interactions (AMMI) were used. Results. The Tetralite II, Bóxer, Bestfor Plus, and Aubade genotypes had the highest green forage yields (7.52-8.34 t.ha.cut $\left.{ }^{-1}\right)$ and dry matter (1.29-1.37 t.ha.cut $\left.{ }^{-1}\right)$ in the three environments studied. With the Eberhart and Russell model, for RFV, these genotypes were classified as the best response in favorable and predictable environments, and by RMS they were also predictable, but Aubade and Bestfor Plus were classified with good response in all environments, and Bóxer and Tetralite II, with better response only in favorable environments. Conclusion. The AMMI model allowed to identify the Pasto municipality as the most favorable environment, and the Bóxer and Tetralite II genotypes as those with the best performing in this environment.

Keywords: Ryegrass, adaptability, AMMI, green fodder.

\section{Introducción}

Las especies del género Lolium tienen una amplia utilización como forraje en la ganadería moderna. El raigrás italiano (Lolium multiflorum) y el inglés (Lolium perenne) son las especies más utilizadas en las zonas templadohúmedas de Europa y Nueva Zelanda, aprovechadas en ensilajes, como forraje verde, en pastoreo y como césped (López, 2009). En la actualidad, estos forrajes representan un componente importante en la alimentación bovina en varias regiones de Colombia, debido principalmente a su buen crecimiento y desarrollo, su elevada productividad, precocidad y calidad nutritiva (Legarda-López, et al., 2015), sin embargo, en el trópico alto de Nariño, una de las principales cuencas lecheras de Colombia (Castrillón, 2014), menos del $20 \%$ del área cultivada con pastos está dedicada a esta gramínea, siendo el kikuyo (Cenchrus clandestinus) el predominante en esta zona, el cual presenta baja productividad por susceptibilidad a algunas plagas, enfermedades y a heladas (AGROSAVIA, 2016); por lo que, es necesario diversificar la oferta forrajera.

Existe poco conocimiento sobre el comportamiento de los cultivares de raigrás que se manejan en Nariño, y no existen estudios de estabilidad que permitan conocer cuáles de esos cultivares que se ofrecen en el mercado se comportan mejor en varias o en una determinada localidad, lo cual afecta la economía de los ganaderos, puesto que corren el riesgo de que los genotipos que llevan a sus fincas no presenten buenos rendimientos. Dado que la respuesta de los genotipos al ser evaluados en distintos ambientes es diferente, se requieren estudios de estabilidad que permitan determinar ese comportamiento diferencial, lo que se conoce como interacción genotipo $\times$ ambiente (Vallejo y Estrada, 2013; Gordón-Mendoza et al., 2006).

Existen diferentes modelos estadísticos que se pueden utilizar para el estudio de la interacción genotipo $\times$ ambiente o la estabilidad fenotípica de genotipos cuando son evaluados en diferentes ambientes (Lozano-del-Río et al., 2009; Flores, 2010; Lozano-Ramírez et al., 2015; Vargas et al., 2016); entre ellos, los modelos propuestos por Eberhart y Russell (1966) y el modelo de efectos principales aditivos e interacciones multiplicativas (AMMI). El objetivo de este trabajo fue determinar la estabilidad fenotípica del rendimiento del forraje verde (RFV) y de la materia seca (RMS) en raigrás, en la cuenca lechera del trópico alto de Nariño, Colombia. 


\section{Materiales y métodos}

\section{Localización}

Entre los años 2016 y 2017, en las localidades de Pasto, Cumbal y Sapuyes, ubicadas en la cuenca lechera del trópico alto de Nariño, Colombia, a altitudes entre 2905 y 3157 msnm, se establecieron tres experimentos, uno en cada localidad, para la evaluación de estabilidad fenotípica de raigrás (Lolium sp.). Los parámetros edafoclimáticos de cada localidad se indican en el Cuadro 1.

Cuadro 1. Ubicación y parámetros edafoclimáticos de las localidades donde se establecieron los experimentos, para la evaluación de estabilidad fenotípica de raigrás (Lolium sp.), en Nariño, Colombia. 2016-2017.

Table 1. Location and edaphoclimatic parameters of the localities where the experiments were established, for the evaluation of phenotypic stability of ryegrass (Lolium sp.), in Nariño, Colombia. 2016-2017.

\begin{tabular}{|c|c|c|c|c|c|c|}
\hline Municipio & Localidades & Coordenadas & $\begin{array}{l}\text { Altitud } \\
\text { (msnm) }\end{array}$ & $\begin{array}{c}\text { Temperatura } \\
\text { media anual } \\
\left({ }^{\circ} \mathrm{C}\right)^{1}\end{array}$ & $\begin{array}{l}\text { Precipitación } \\
\text { media anual } \\
(\mathbf{m m})^{1}\end{array}$ & Textura de suelo \\
\hline Pasto & $\begin{array}{l}\text { C.I. Obonuco } \\
\text { AGROSAVIA* }\end{array}$ & $\begin{array}{l}\text { N } 01^{\circ} 11^{\prime} 4,13^{\prime \prime} \\
\text { W } 77^{\circ} 19^{\prime} 0,19^{\prime \prime}\end{array}$ & 2905 & 11,0 & 1273 & Franco arenosos \\
\hline Cumbal & $\begin{array}{l}\text { Vereda San José } \\
\text { Finca de productor }\end{array}$ & $\begin{array}{l}\text { N } 00^{\circ} 53^{\prime} 12,1^{\prime \prime} \\
\text { W } 77^{\circ} 48^{\prime} 8,06^{\prime \prime}\end{array}$ & 3210 & 10,5 & 1050 & Franco arcillosos \\
\hline Sapuyes & $\begin{array}{l}\text { Granja Exp. Chimangual } \\
\text { Universidad de Nariño }\end{array}$ & $\begin{array}{l}\text { N } 01^{\circ} 02^{\prime} 6,55^{\prime \prime} \\
\text { W } 77^{\circ} 45^{\prime} 3,88^{\prime \prime}\end{array}$ & 3157 & 10,2 & 1061 & $\begin{array}{l}\text { Franco areno- } \\
\text { gravilloso }\end{array}$ \\
\hline
\end{tabular}

${ }^{1}$ Fuente/Source: Climate-Data.org, 2018.

* Centro de Investigación Obonuco, Corporación Colombiana de Investigación Agropecuaria / * Obonuco Research Center, Corporación Colombiana de Investigación Agropecuaria.

En los tres experimentos se realizó el mismo manejo. Para la preparación del suelo se efectuaron dos pases de cincel vibratorio y dos pases de rastra pesada. La siembra de los genotipos de raigrás se realizó al voleo, a una densidad de $50 \mathrm{~kg} \cdot \mathrm{ha}^{-1}$, según recomendación de la casa comercial. La fertilización se realizó manualmente con $100 \mathrm{~kg} \mathrm{~N} \cdot \mathrm{ha}^{-1}$, $75 \mathrm{~kg} \mathrm{P}_{2} \mathrm{O}_{5} \cdot \mathrm{ha}^{-1}, 30 \mathrm{~kg} \mathrm{~K}_{2} \mathrm{O} \cdot \mathrm{ha}^{-1}$ y $12 \mathrm{~kg} \mathrm{Mg} \cdot \mathrm{ha}^{-1}$, en dos aplicaciones por año, una a la siembra y otra al sexto mes.

\section{Materiales evaluados}

Se evaluaron diez genotipos de raigrás (Cuadro 2), dos diploides y ocho tetraploides, considerando que eran los más comercializados, por disponibilidad de semilla, en la zona lechera de Nariño, y teniendo en cuenta las características de adaptación al clima y suelos de trópico alto.

\section{Diseño experimental}

En cada localidad, los experimentos se establecieron bajo un diseño de bloques completos al azar (DBCA) con cuatro repeticiones y diez tratamientos (cada genotipo constituyó un tratamiento). Cada unidad experimental tuvo un área de $6 \mathrm{~m}^{2}$. 
Cuadro 2. Genotipos de raigrás (Lolium sp.) evaluados en el trópico alto de Nariño, Colombia. 2016-2017.

Table 2. Genotypes of ryegrass (Lolium sp.) evaluated in the high tropic of Nariño, Colombia. 2016-2017.

\begin{tabular}{ccc}
\hline Genotipo & Nombre científico & Ploidía \\
\hline Ohau & Lolium hybridum & Tetraploide \\
Boxer & Lolium hybridum & Tetraploide \\
Samson & Lolium perenne & Diploide \\
Aubade & Lolium hybridum & Tetraploide \\
Bestfor Plus & Lolium multiflorum & Tetraploide \\
Magnum & Lolium multiflorum & Tetraploide \\
Tetralite II & Lolium hybridum & Tetraploide \\
Bison & Lolium multiflorum & Tetraploide \\
Conquistador & Lolium perenne & Diploide \\
Angus & Lolium westerwoldicum & Tetraploide \\
\hline
\end{tabular}

\section{Variables evaluadas}

\section{Rendimiento de forraje verde $\left(t . h a^{-1}\right)$}

En cada localidad, después del corte de uniformización (noventa días después de la siembra), se realizaron ciclos de corte en cada experimento: en la localidad de Pasto, dos en época de lluvias y dos en época seca; en Cumbal uno en lluviosa y dos en seca, y en Sapuyes uno en lluviosa y tres en seca. En cada ciclo se evaluaron cuatro frecuencias de corte, a los 25, 30, 35 y 40 días. Las muestras de forraje se obtuvieron en $1 \mathrm{~m}^{2}$ y a $5 \mathrm{~cm}$ de altura sobre el suelo.

\section{Rendimiento de materia seca $\left(t . h a^{-1}\right)$}

De cada muestra de forraje verde, se tomó una submuestra de $500 \mathrm{~g}$ y se secó a una temperatura de $70{ }^{\circ} \mathrm{C}$ durante $48 \mathrm{~h}$ (CIAT, 1982). Los datos obtenidos permitieron calcular materia seca (\%) y rendimiento de materia seca.

\section{Análisis estadístico de resultados}

Previo al análisis de varianza, se realizó una prueba de homogeneidad de varianza y normalidad de Kolmogorov - Smirnov, donde se observó que los datos se ajustaron a la curva normal ( $>0,05)$. Para clasificar los genotipos por su estabilidad fenotípica, se trabajó con el rendimiento de forraje verde y de materia seca a través de las tres localidades, mediante el modelo de Eberhart y Russell (1966) y el modelo de análisis de efectos principales aditivos e interacciones multiplicativas (AMMI), utilizando el software SAS 9.4.

En el modelo propuesto por Eberhart y Russell (1966), se realizó un análisis de regresión lineal del promedio de cada genotipo sobre el índice ambiental de cada localidad (diferencia entre rendimiento promedio de cada localidad y el promedio de todos los genotipos en las tres localidades donde se realizó la evaluación), con el fin de estimar la estabilidad. El modelo AMMI combinó el análisis de varianza para los efectos principales de genotipos y ambientes con el análisis de componentes principales de la interacción genotipo $\times$ ambiente $(\mathrm{G} \times \mathrm{A})$; los resultados 
se graficaron en biplots y se colocaron tanto los efectos principales como los efectos de la interacción para los genotipos y los ambientes.

\section{Resultados}

\section{Análisis de estabilidad fenotípica - Modelo de Eberhart y Russell}

Hubo altas significancias $(\mathrm{p}<0,01)$ para ambientes, genotipos y la interacción genotipo $\times$ ambiente, en el rendimiento de forraje verde y de materia seca para los genotipos de raigrás estudiados, las cuales permitieron separarlos por sus características de adaptabilidad y estabilidad, con base en el análisis de varianza de la regresión lineal.

\section{Adaptabilidad y estabilidad por rendimientos de forraje verde - Modelo de Eberhart y Russell}

Con base en los criterios de clasificación de Eberhart y Russell, para rendimientos de forraje verde (t.ha.corte ${ }^{-1}$ ), se presentó un comportamiento diferencial entre genotipos por efecto de los ambientes (Cuadro 3), donde: Aubade $(8,34)$, Bestfor Plus $(8,30)$, Bóxer $(7,61)$ y Tetralite II $(7,52)$ se clasificaron con mejor respuesta en ambientes favorables y fueron predecibles $\left(b_{i}>1 ; S^{2} d i=0\right)$ (parte superior de la Figura 1). Los genotipos Conquistador $(6,69)$, Magnum $(6,60)$ y Angus $(6,14)$, se clasificaron con buena respuesta en todos los ambientes y fueron predecibles $\left(b_{i}=1 ; S^{2} d i=0\right)$ (parte media de la Figura 1, entre líneas paralelas azules). Dentro del grupo con mejor respuesta en ambientes desfavorables y predecibles $\left(b_{i}<1 ; S^{2} d i=0\right)$ estuvieron Ohau $(6,29)$, Bison $(5,53)$ y Samson $(5,19)$ (parte inferior de la Figura 1).

Cuadro 3. Clasificación de diez genotipos de raigrás (Lolium sp.), por rendimiento de forraje verde (t.ha.corte ${ }^{-1}$ ), según los criterios de estabilidad de Eberhart y Russell. Nariño, Colombia. 2016-2017.

Table 3. Classification of ten ryegrass (Lolium sp.) genotypes by green forage yield (t.ha.cut ${ }^{-1}$ ), according to the stability criteria of Eberhart and Russell. Nariño, Colombia. 2016-2017.

\begin{tabular}{|c|c|c|c|c|c|}
\hline Genotipos & $\begin{array}{c}\text { Promedio } \\
\left(\mathbf{b}_{\mathbf{o}}\right)\end{array}$ & $\begin{array}{l}\text { Coeficiente } \\
\text { Regresión } \\
\left(\mathbf{b}_{\mathrm{i}}\right)\end{array}$ & $\begin{array}{l}\text { CM desviación } \\
\text { regresión } \\
\left(\mathbf{S}^{2} \mathbf{d i}\right)\end{array}$ & $\begin{array}{c}\text { Coeficiente } \\
\text { determinación } \\
\left(\mathbf{R}^{2}\right)\end{array}$ & Clasificación genotipos \\
\hline Aubade* & 8,34 & 1,09 & $-3,96$ & 0,9670 & Mejor respuesta en ambientes favorables y predecibles \\
\hline Bestfor Plus*** & 8,30 & 1,08 & $-3,86$ & 0,9642 & Mejor respuesta en ambientes favorables y predecibles \\
\hline Boxer* & 7,61 & 1,18 & $-5,29$ & 0,9986 & Mejor respuesta en ambientes favorables y predecibles \\
\hline Tetralite II* & 7,52 & 1,17 & $-4,71$ & 0,9866 & Mejor respuesta en ambientes favorables y predecibles \\
\hline Conquistador** & 6,69 & 1,00 & $-1,83$ & 0,9080 & Buena respuesta en todos los ambientes y predecible \\
\hline Magnum*** & 6,60 & 1,04 & $-5,33$ & 0,9992 & Buena respuesta en todos los ambientes y predecible \\
\hline Ohau* & 6,29 & 0,90 & $-5,06$ & 0,9894 & $\begin{array}{l}\text { Mejor respuesta en ambientes desfavorables y } \\
\text { predecible }\end{array}$ \\
\hline Angus $* * * *$ & 6,14 & 1,05 & $-2,82$ & 0,9378 & Buena respuesta en todos los ambientes y predecible \\
\hline Bison*** & 5,53 & 0,77 & $-4,34$ & 0,9531 & $\begin{array}{l}\text { Mejor respuesta en ambientes desfavorables y } \\
\text { predecible }\end{array}$ \\
\hline Samson** & 5,19 & 0,70 & $-4,97$ & 0,9772 & $\begin{array}{l}\text { Mejor respuesta en ambientes desfavorables y } \\
\text { predecible }\end{array}$ \\
\hline
\end{tabular}

* L. hybridum. ** L. perenne. *** L. multiflorum. **** Lolium westerwoldicum. 


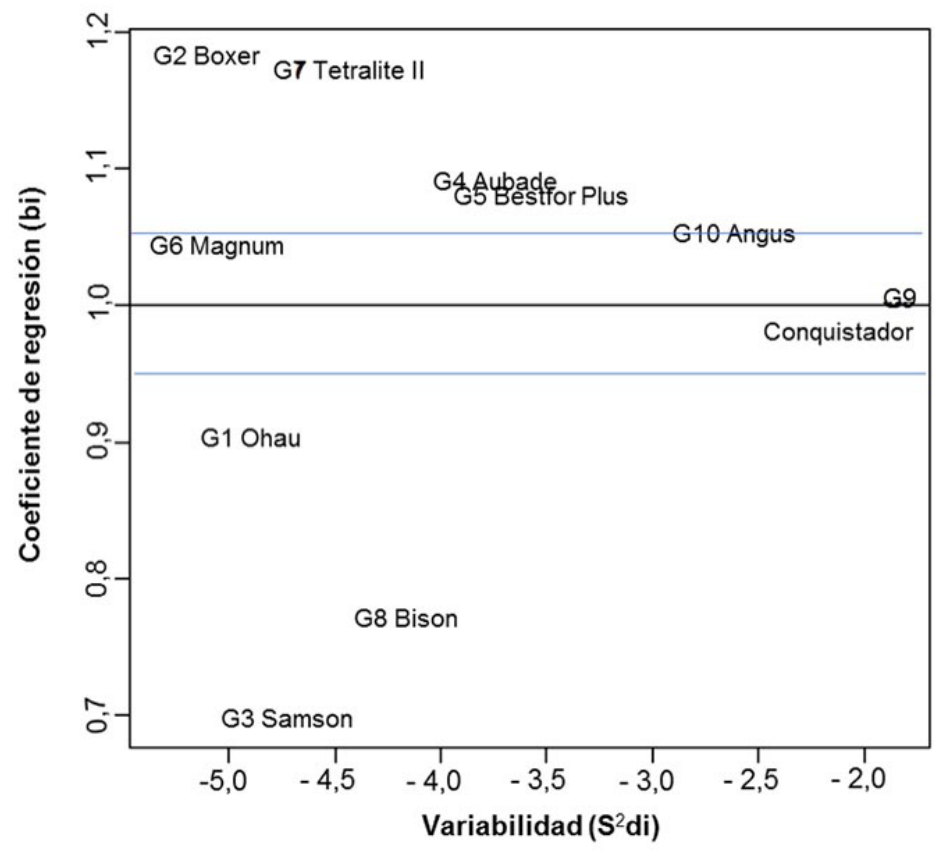

Figura 1. Coeficiente de regresión (bi) y cuadrado medio de desviación de la regresión o variabilidad (S²di) de diez genotipos de raigrás (Lolium sp.), evaluados por su estabilidad fenotípica mediante Eberhart y Russell, con base en rendimiento de forraje verde en tres ambientes. Nariño, Colombia. 2016-2017.

Figure 1. Regression coefficient (bi) and mean squared deviation of the regression or variability ( $\mathrm{S}^{2} \mathrm{di}$ ) of ten ryegrass (Lolium sp.) genotypes, evaluated for their phenotypic stability by Eberhart and Russell, based on green forage yield in three environments. Nariño, Colombia. 2016-2017.

En este trabajo, todas las desviaciones de la regresión fueron negativas y, por tanto, iguales a cero clasificando a todos los genotipos con estabilidad predecible (Cuadro 3). Además, la proporción de la varianza total de las variables rendimiento de forraje verde y de materia seca fueron explicadas por las regresiones con valores superiores a 0,9079, mediante el coeficiente de determinación $\left(\mathrm{R}^{2}\right)$ y reflejando la bondad del ajuste del modelo.

\section{Adaptabilidad y estabilidad por rendimientos de materia seca - Modelo de Eberhart y Russell}

Con base en el rendimiento de la materia seca (t.ha.corte ${ }^{-1}$ ) (Cuadro 4), todos los genotipos fueron predecibles $\left(\mathrm{S}^{2} \mathrm{di}=0\right)$. Bestfor Plus $(1,37)$, Aubade $(1,35)$, Angus $(1,12)$ y Ohau $(1,12)$, se clasificaron con buena respuesta en todos los ambientes $\left(b_{i}=1\right)$ (parte media de la Figura 2). Bóxer $(1,31)$ y Tetralite II $(1,29)$, mostraron mejor respuesta en ambientes favorables $\left(b_{i}>1\right)$ (parte superior de la Figura 2). Los genotipos con mejor respuesta en ambientes desfavorables $\left(b_{i}<1\right)$ fueron Conquistador $(1,17)$, Magnum $(1,16)$, Bison $(1,07)$ y Samson $(1,03)$ (parte inferior de la Figura 2).

\section{Análisis de estabilidad por rendimientos de forraje verde y de materia seca - Modelo AMMI}

El análisis de varianza para rendimientos de forraje verde y de materia seca por AMMI, mostró altas significancias $(\mathrm{p}<0,01)$ para genotipos, ambientes y la interacción genotipo $\times$ ambiente $(\mathrm{G} \times \mathrm{A})$, lo cual concuerda con lo encontrado mediante el modelo de Eberhart y Russell. 
Cuadro 4. Clasificación de diez genotipos de raigrás (Lolium sp.), por rendimiento de materia seca (t.ha.corte-1), según los criterios de estabilidad de Eberhart y Russell. Nariño, Colombia. 2016-2017.

Table 4. Classification of ten ryegrass (Lolium sp.) genotypes, by yield of dry matter (t.ha.cut ${ }^{-1}$ ), according to the stability criteria Eberhart and Russell. Nariño, Colombia. 2016-2017.

\begin{tabular}{|c|c|c|c|c|c|}
\hline Genotipos & $\begin{array}{c}\text { Promedio } \\
\left(\mathbf{b}_{\mathbf{o}}\right)\end{array}$ & $\begin{array}{c}\text { Coeficiente } \\
\text { Regresión } \\
\left(b_{i}\right)\end{array}$ & $\begin{array}{c}\text { CM desviación } \\
\text { regresión } \\
\left(\mathbf{S}^{2} \mathbf{d i}\right) \\
\end{array}$ & $\begin{array}{c}\begin{array}{c}\text { Coeficiente } \\
\text { determinación }\end{array} \\
\left(\mathbf{R}^{2}\right)\end{array}$ & Clasificación genotipos \\
\hline Bestfor Plus*** & 1,37 & 1,03 & $-0,08$ & 0,9673 & Buena respuesta en todos los ambientes y predecible \\
\hline Aubade* & 1,35 & 0,96 & $-0,09$ & 0,9711 & Buena respuesta en todos los ambientes y predecible \\
\hline Bóxer* & 1,31 & 1,17 & $-0,11$ & 0,9997 & Mejor respuesta en ambientes favorables y predecible \\
\hline Tetralite II* & 1,29 & 1,20 & $-0,09$ & 0,9863 & Mejor respuesta en ambientes favorables y predecible \\
\hline Conquistador** & 1,17 & 0,93 & $-0,07$ & 0,9351 & Mejor respuesta en ambientes desfavorables y predecible \\
\hline Magnum $* * *$ & 1,16 & 0,94 & $-0,11$ & 0,9953 & Mejor respuesta en ambientes desfavorables y predecible \\
\hline Angus**** & 1,12 & 1,02 & $-0,07$ & 0,9453 & Buena respuesta en todos los ambientes y predecible \\
\hline Ohau* & 1,12 & 1,01 & $-0,11$ & 0,9992 & Buena respuesta en todos los ambientes y predecible \\
\hline Bison*** & 1,07 & 0,82 & $-0,09$ & 0,9575 & Mejor respuesta en ambientes desfavorables y predecible \\
\hline Samson** & 1,03 & 0,92 & $-0,11$ & 0,9969 & Mejor respuesta en ambientes desfavorables y predecible \\
\hline
\end{tabular}

* L. hybridum. ${ }^{* *}$ L. perenne. *** L. multiflorum. **** Lolium westerwoldicum.

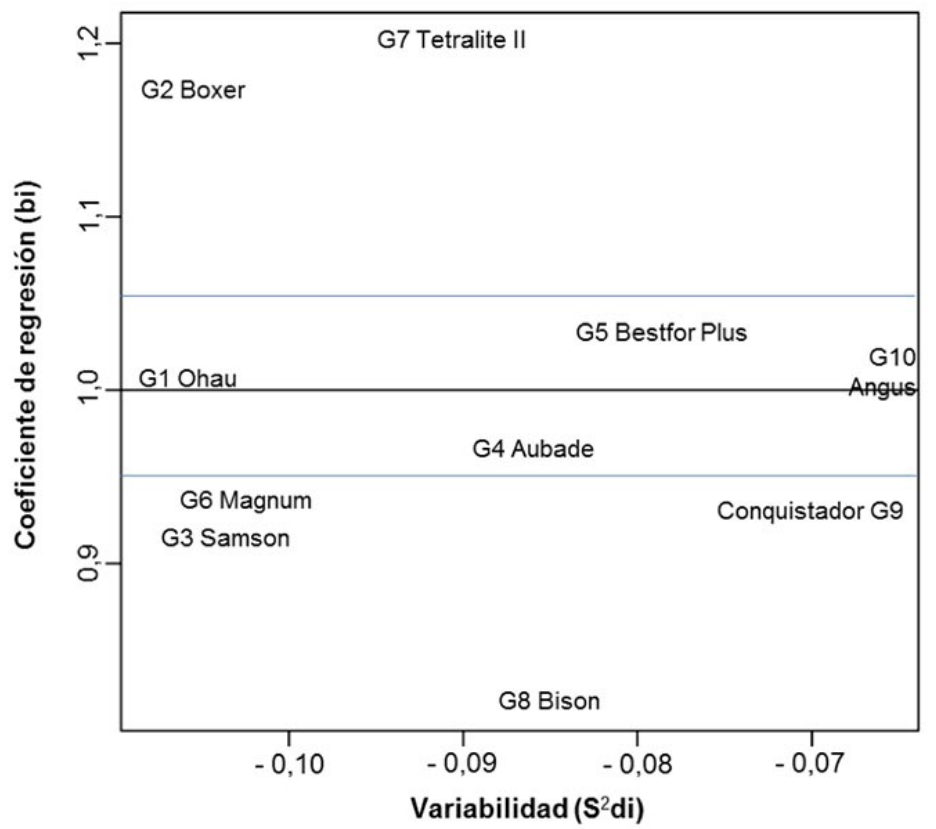

Figura 2. Coeficiente de regresión (bi) y cuadrado medio de desviación de la regresión o variabilidad ( $\mathrm{S}^{2}$ di) de diez genotipos de raigrás (Lolium sp.), evaluados por su estabilidad fenotípica mediante Eberhart y Russell, con base en rendimiento de materia seca en tres ambientes. Nariño, Colombia. 2016-2017.

Figure 2. Regression coefficient (bi) and mean squared deviation of the regression or variability ( $\left.\mathrm{S}^{2} \mathrm{di}\right)$ of ten ryegrass (Lolium sp.) genotypes, evaluated for their phenotypic stability by Eberhart and Russell, based on dry matter yield in three environments. Nariño, Colombia. 2016-2017. 


\section{Estabilidad por rendimiento de forraje verde - Modelo AMMI}

Mediante el modelo AMMI, aplicado a los valores de rendimiento de forraje verde de los genotipos de raigrás, se generó una figura biplot en dos dimensiones que corresponden a los dos primeros componentes principales, la cual muestra el efecto de la interacción genotipo $\times$ ambiente (Figura 3). El primer componente principal (CP1) explicó el 59,04\% de la varianza total y el segundo (CP2) el 40,67 \% (Figura 3). En total, los dos primeros componentes explicaron el 99,71\% de la variabilidad total.

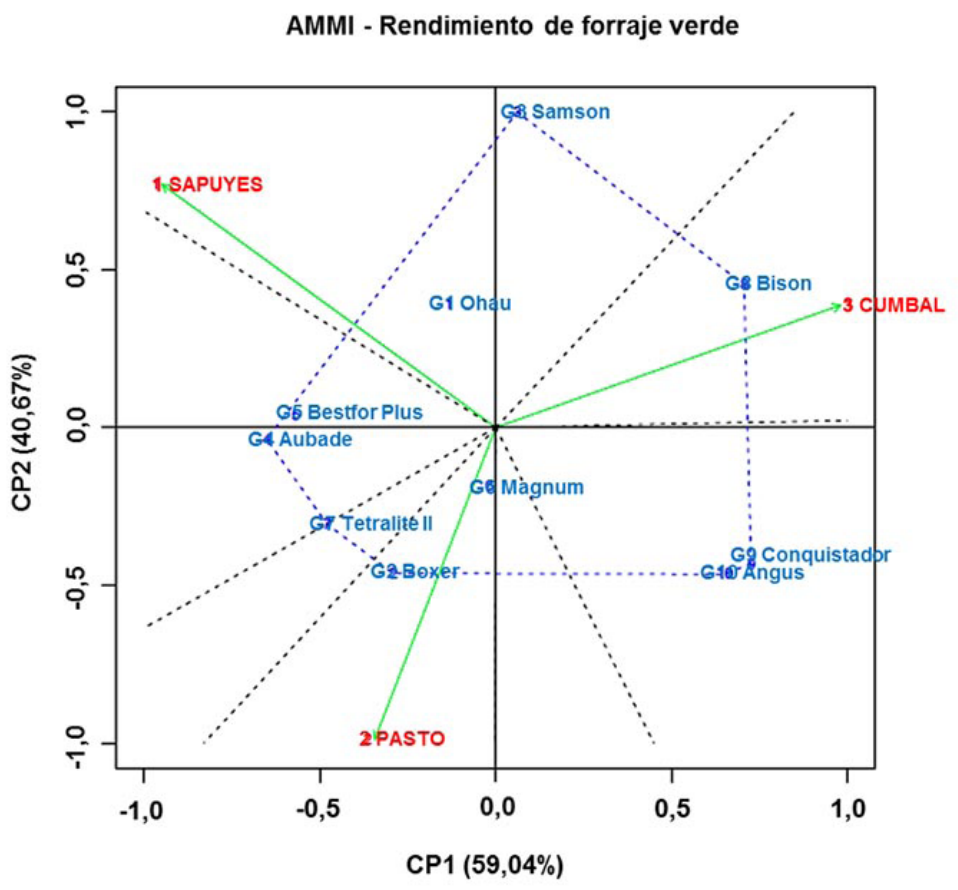

Figura 3. Biplot AMMI para rendimiento de forraje verde de genotipos de raigrás (Lolium sp.) y ambientes de evaluación con respecto a los dos primeros ejes de componentes principales (CP1 y CP2). Nariño, Colombia. 2016-2017.

Figure 3. AMMI biplot for green forage yield of ryegrass (Lolium sp.) genotypes, and evaluation environments in relation to the first two axes of principal components (CP1 and CP2). Nariño, Colombia. 2016-2017.

El biplot AMMI (Figura 3), mostró la diferencia entre ambientes cuando se analizó la variable rendimiento de forraje verde (t.ha.corte ${ }^{-1}$ ). El marcador ambiental más cercano al origen fue Pasto $(11,55)$, siendo el ambiente más estable. Cumbal $(5,17)$ y Sapuyes $(3,74)$ estuvieron más distantes del origen y se clasificaron como ambientes inestables.

Los genotipos Magnum $(6,60)$ y Ohau $(6,29)$, más cercanos al origen, mostraron la mayor estabilidad cuando se analizaron por rendimiento de forraje verde (t.ha.corte ${ }^{-1}$ ) (Figura 3), además, los genotipos Aubade $(8,34)$, Bestfor Plus $(8,30)$, Bóxer $(7,61)$ y Tetralite II $(7,52)$, alejados del origen, se localizaron cerca del vector de Pasto, por lo cual, se consideraron como inestables, pero con buen comportamiento en cuanto a rendimiento de forraje verde en este ambiente. Los genotipos que mejor se asociaron con el ambiente Cumbal fueron Bison $(5,53)$, Conquistador $(6,69)$ y Angus $(6,14)$. En Sapuyes, el ambiente más desfavorable, los genotipos más asociados fueron Aubade $(8,34)$, Bestfor Plus $(8,30)$, Ohau $(6,29)$ y Samson $(5,19)$. 
Los genotipos Bestfor Plus $(8,30)$ y Aubade $(8,34)$, se comportaron en forma similar, por su proximidad (Figura 3 ), y mostraron los mejores rendimientos; mientras que, Conquistador $(6,69)$ y Angus $(6,14)$, se comportaron de forma similar, pero con rendimientos intermedios en comparación con los demás genotipos.

Los ambientes se presentaron como vectores que se originaron en la coordenada $(0,0)$ del biplot y se extienden hasta el marcador correspondiente. En esta investigación, teniendo en cuenta la variable rendimiento de forraje verde, los tres ambientes tuvieron asociación negativa, dado que los vectores Pasto, Cumbal y Sapuyes formaron ángulo obtuso entre ellos (Figura 3).

\section{Estabilidad por rendimientos de materia seca - Modelo AMMI}

En forma similar al análisis realizado para rendimiento de forraje verde, se generó un biplot que mostró el efecto de la interacción genotipo $\times$ ambiente (Figura 4), en donde el primer componente principal (CP1) explicó el $73,31 \%$ de la varianza total y el segundo (CP2) el 26,29\%. En total los dos primeros componentes explicaron el $99,60 \%$ de la variabilidad total.

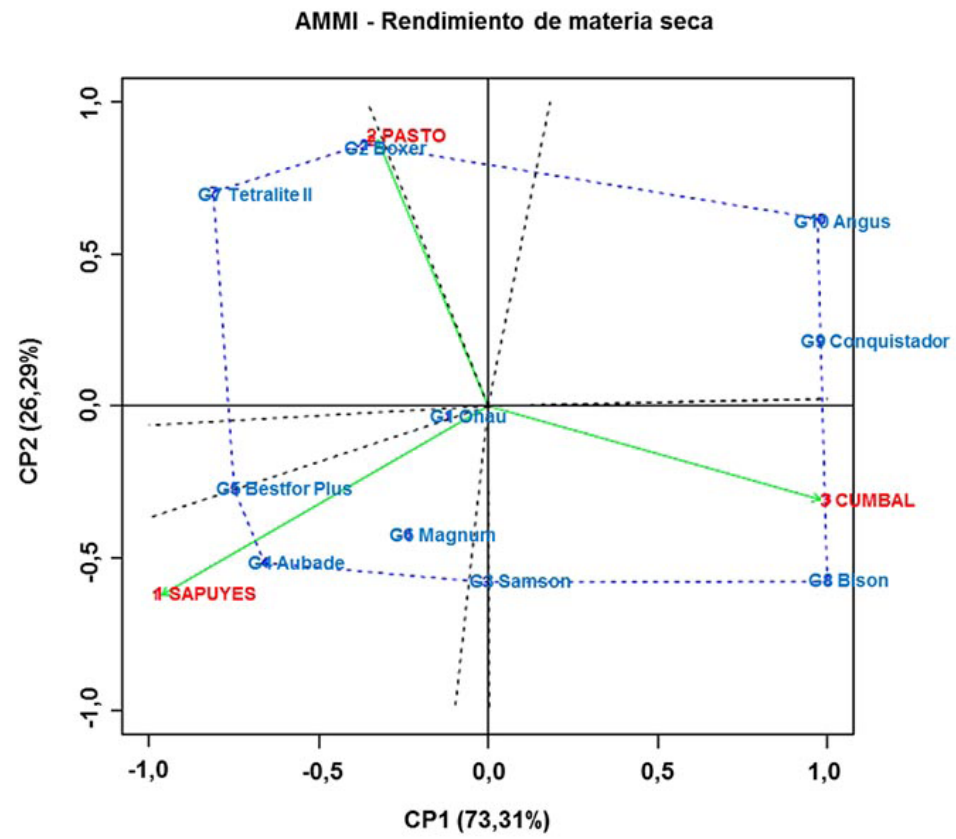

Figura 4. Biplot AMMI para rendimiento de materia seca de genotipos de raigrás (Lolium sp.) y ambientes de evaluación con respecto a los dos primeros ejes de componentes principales (CP1 y CP2). Nariño, Colombia. 2016-2017.

Figure 4. AMMI biplot for dry matter yield of ryegrass (Lolium sp.) genotypes, and evaluation environments in relation to the first two axes of principal components (CP1 and CP2). Nariño, Colombia. 2016-2017.

El biplot AMMI para rendimiento (t.ha.corte ${ }^{-1}$ ) de materia seca (Figura 4), mostró diferencias entre ambientes. Pasto $(1,88)$ fue el más cercano al origen y, por tanto, el ambiente más estable; mientras que, Cumbal $(0,97)$ y Sapuyes $(0,75)$, más alejados del origen fueron ambientes inestables. 
El genotipo Ohau fue el más cercano al origen y mostró la mayor estabilidad cuando se analizó por rendimiento de materia seca. Los genotipos que mejor se asociaron con Pasto fueron Bóxer y Tetralite II. En Cumbal fueron Bison, Conquistador y Angus, mientras que Aubade, Bestfor Plus, Magnum y Samson se localizaron cerca del vector de Sapuyes, considerados estos últimos como poco estables, pero con buen comportamiento en cuanto a rendimiento de materia seca en este ambiente (Figura 4).

\section{Discusión}

En el análisis de varianza, los efectos altamente significativos encontrados entre ambientes, con base en las variables de rendimiento de forraje verde y de materia seca, confirmaron las diferencias entre las tres localidades, provocadas sobre todo por el tipo de suelo, altitud, temperatura y precipitación de cada una (Cuadro 1). De igual manera, para el caso de los genotipos, se observó un rendimiento diferencial de acuerdo con su genética y potencial de producción, mientras que el efecto estimado en la interacción demostró la respuesta diferencial de los genotipos al evaluarse en los distintos ambientes. También se encontraron altos efectos ambientales, de genotipo y de la interacción genotipo $\times$ ambiente en una investigación sobre variación genética para la producción de semillas, en nueve genotipos de raigrás perenne y doce ambientes (Elgersma, 1990). Resultados similares encontraron Ibáñez et al. (2001) en su estudio, en Argentina, con diecinueve genotipos de un pasto utilizado para alimentación bovina, conocido como hierba de amor (Eragrostis curvula), donde se trabajó en seis ambientes.

En el análisis de estabilidad fenotípica, por rendimiento de forraje verde y de materia seca, mediante el método de Eberhart y Russell, las medias de los genotipos se ajustaron a la línea de regresión y correlacionaron significativamente con los índices ambientales; por tanto, se pueden predecir con mayor confiabilidad los rendimientos que lograrán en ambientes favorables y desfavorables. Esto se corrobora porque los valores de las desviaciones $\left(\mathrm{S}^{2} \mathrm{di}\right)$ alrededor de la recta de regresión fueron negativos y, por tanto, iguales a cero, teniendo en cuenta lo mencionado por Vallejo y Estrada (2013), quienes afirman que: "valores negativos de las desviaciones de la regresión se interpretan como estimaciones de un parámetro poblacional igual a cero"; cabe resaltar que al mejorador le interesan variedades que tengan el valor $\mathrm{S}^{2}$ di lo más próximo a cero.

Con base en los criterios de clasificación de Eberhart y Russell, tanto para rendimiento de forraje verde como para materia seca, se presentó un comportamiento diferencial entre genotipos por efecto de los ambientes, formándose tres grupos; por una parte, los que presentaron su mejor respuesta en ambientes favorables, que para este caso fue la localidad de Pasto (con el mejor índice ambiental) por tener las mejores condiciones edafoclimáticas. Por otra parte, los que presentaron su mejor respuesta en ambientes desfavorables (Cumbal y Sapuyes) y, finalmente, los que mostraron mayor grado de estabilidad y presentaron buena respuesta en las todas las localidades o ambientes. En el último grupo estuvieron los genotipos Conquistador, Magnum y Angus en la variable rendimiento de forraje verde, aunque estos tuvieron rendimientos inferiores a la media general $\left(<6,82\right.$ t.ha.corte $\left.{ }^{-1}\right)$; mientras que, para el caso de materia seca, los genotipos Bestfor Plus y Aubade, además de presentar mayor estabilidad, superaron a la media general ( $>1,19$ t.ha. corte $^{-1}$ ) en un 15,12 y 13,44 \%, respectivamente. Estos últimos genotipos no estuvieron afectados en forma significativa por las condiciones de cada localidad o ambiente, por lo cual podrían servir para una validación de su respuesta a nivel semi comercial en las tres localidades. Los mecanismos genéticos que operan en la manifestación de la estabilidad espacial son la diversidad genética, heterocigosis, plasticidad genotípica y poliploidía (Vega, 1988).

Con el modelo AMMI, la suma de la variabilidad explicada por los dos primeros componentes principales, en las dos variables de estudio, alcanzó más del $99 \%$, superior a lo encontrado por Méndez et al. (2014) en un estudio de interacción genotipo $\times$ ambiente en cultivares de Lolium multiflorum, donde se alcanzó el 85,3\% cuando se evaluó el rendimiento de materia seca. Esto, posiblemente, se atribuye a las diferencias en el número de genotipos y localidades, con distintas condiciones ambientales, evaluados en cada investigación. 
El modelo AMMI, como lo afirma Mejía (2014), permite identificar genotipos con mejor comportamiento en un ambiente específico, identificar el ambiente más apropiado para un determinado genotipo y la comparación de cualquier par de genotipos en un ambiente.

Para rendimiento de forraje verde y materia seca fue posible evidenciar diferencias entre ambientes; para las dos variables el marcador más cercano al origen fue Pasto y, por tanto, el más estable, contribuyendo poco a la interacción (Namorato et al., 2009); mientras que, Cumbal y Sapuyes fueron los ambientes con mayor variación y de alguna manera, los desfavorables. Esta clasificación de ambientes concuerda con la encontrada con el método Eberhart y Russell, teniendo en cuenta los índices ambientales, donde Pasto fue muy favorable $(4,73)$, mientras que, Cumbal $(-1,65)$ y Sapuyes $(-3,08)$ fueron ambientes desfavorables.

Con respecto a los cultivares, el AMMI también permitió identificar genotipos que presentaron mayor grado de estabilidad, denotada por su cercanía al origen de coordenadas de los biplots (Frutos, 2011). Los genotipos Magnum $(6,60)$ y Ohau $(6,29)$, para rendimiento de forraje verde y este último también para materia seca, mostraron una menor respuesta a los cambios ambientales, sin embargo, el rendimiento promedio de estos en los tres ambientes no fue superior al promedio general $(6,82)$. Los genotipos que se comportaron mejor en un ambiente determinado, cuando se analizaron por rendimiento de forraje verde (t.ha.corte $\left.{ }^{-1}\right)$, fueron Aubade $(13,33)$, Bestfor Plus $(13,24)$, Bóxer $(13,17)$ y Tetralite II $(12,95)$, en la localidad de Pasto; en Cumbal Conquistador $(3,59)$, Angus $(3,18)$ y Bison $(3,48)$, este último por estar en un vértice y alejado del origen aportó en forma importante a la interacción; y en Sapuyes Bestfor Plus $(4,20)$, Aubade $(4,24)$, Ohau $(3,16)$ y Samson $(2,66)$. Con respecto a materia seca, los genotipos Bóxer y Tetralite II presentaron un mejor comportamiento en la localidad de Pasto; Magnum, Samson, Aubade y Bestfor Plus $(1,37)$ en Sapuyes, y Bison en Cumbal. Al igual que en el modelo de Eberhart y Russell, las respuestas diferenciales encontradas en el comportamiento de los genotipos pueden atribuirse a las diferencias en los períodos de evaluación, los ambientes de evaluación, los limitantes climáticos como la cantidad de lluvia, principalmente, y la constitución genética de los raigrases evaluados.

En general, y comparando con el método de Eberhart y Russell, los genotipos que presentaron valores significativos para el coeficiente de regresión $\left(b_{i}<1\right.$ y $\left.b_{i}>1\right)$, fueron los que presentaron mayor distanciamiento del origen de los ejes, lo cual coincide con lo encontrado por Damba (2008), en un estudio de estabilidad en genotipos de yuca.

Se identificaron genotipos con comportamientos similares en cuanto a las variables de respuesta evaluadas, indicadas por las proximidades entre dos o más genotipos (Frutos, 2011), como el caso de Bestfor Plus (8,30 t.ha. corte $^{-1}$ ) y Aubade (8,34 tha.corte $\left.{ }^{-1}\right)$ destacados por los mejores rendimientos de forraje verde, sin embargo, no presentaron adaptabilidad general (igual comportamiento en todos los ambientes), sino que se aproximaron más al ambiente favorable.

Los ambientes cercanos entre sí, dado por el ángulo agudo entre sus vectores (Figuras 3 y 4), indicaron asociación ambiental positiva (Ibáñez et al., 2006). La ausencia de asociación entre ambientes se dio por el ángulo recto entre vectores y la asociación negativa por el ángulo obtuso. En el presente estudio, considerando las dos variables de respuesta (Figuras 3 y 4), se pudo observar una asociación ambiental negativa o patrones distintos de respuesta en el comportamiento relativo de un conjunto de genotipos, debido en gran parte a las diferentes condiciones edafoclimáticas de cada localidad en las que se establecieron los genotipos evaluados.

\section{Conclusiones}

Mediante el modelo Eberhart y Russell se clasificó a los genotipos Bestfor Plus, Aubade, Boxer y Tetralite II, con mejor comportamiento en rendimiento de forraje verde, como genotipos con mejor respuesta en ambientes favorables y estabilidad predecible, indicando un tipo de estabilidad específica. Por rendimiento de materia seca, 
los genotipos Bestfor Plus y Aubade, se clasificaron como genotipos con buena respuesta en todos los ambientes y predecibles, demostrando un tipo de adaptabilidad general y permitiendo recomendarlos como una alternativa forrajera para las tres localidades. El modelo AMMI indicó que la localidad de Pasto fue el ambiente más favorable, en donde se destacaron por los más altos rendimientos de forraje verde (t.ha.corte $\left.{ }^{-1}\right)$ los genotipos Aubade, Bestfor Plus, Bóxer y Tetralite II, indicando su gran potencial productivo en esta localidad.

\section{Agradecimientos}

Los autores expresan sus agradecimientos a la Corporación Colombiana de Investigación Agropecuaria (AGROSAVIA), a la Sociedad de Agricultores y Ganaderos de Nariño (SAGAN) y a la Universidad de Nariño por haber permitido llevar a cabo esta investigación a través del macroproyecto denominado "Mejoramiento de la oferta forrajera, optimización de sistemas de alimentación y aseguramiento de la calidad e inocuidad de la leche en el trópico alto del departamento de Nariño" con recursos del Sistema General de Regalías.

\section{Literatura citada}

AGROSAVIA (Corporación Colombiana de Investigación Agropecuaria). 2016. Mejoramiento de la oferta forrajera, optimización de sistemas de alimentación y aseguramiento de la calidad e inocuidad de la leche en el trópico alto del departamento de Nariño. AGROSAVIA, Bogotá, COL.

Castrillón, D. 2014. Informe: Cuencas lecheras, motores de la producción nacional. Federación Colombiana de Ganaderos (Fedegán), COL. http://www.fedegan.org.co/noticias/informe-cuencas-lecheras-motores-de-la-produccion-nacional (consultado 07 abr. 2018).

Climate-Data.Org. 2018. Clima: Pasto, Cumbal y Sapuyes. Climate-Data.org https://es.climate-data.org/ (consultado 15 mar. 2018).

CIAT. 1982. Manual para la evaluación agronómica, red internacional de evaluación de pastos tropicales. CIAT, Cali, COL.

Damba, G. 2008. Evaluación de métodos para análisis de estabilidad en diferentes ambientes en genotipos de yuca (Manihot esculenta Crantz.). Tesis MSc., Universidad Nacional de Colombia, COL.

Eberhart, S., and W. Russell. 1966. Stability parameters for comparing varieties. Crops Sci. 6:36-40. doi:10.2135/cropsci1966 .0011183 X000600010011x

Elgersma, A. 1990. Genetic variation for seed yield in perennial ryegrass (Lolium perenne L.). Plant Breed. 105:117-125. doi:10.1111/j.1439-0523.1990.tb00464.x

Flores, D. 2010. Comparación de criterios de selección de híbridos experimentales de sorgo para grano (Sorghum bicolor L. Moench.) para su liberación a la producción comercial. Tesis Ph.D., Universidad Autónoma de Nuevo León, Nuevo León, MEX.

Frutos, M. 2011. Interacción genotipo - ambiente: GGE biplot y modelos AMMI. Tesis MSc., Universidad de Salamanca, ESP.

Gordón-Mendoza R., I. Camargo-Buitrago, J. Franco-Barrera, y A. González-Saavedra. 2006. Evaluación de la adaptabilidad y estabilidad de 14 híbridos de maíz, Azuero, Panamá. Agron. Mesoam. 17:189-199. doi:10.15517/am.v17i2.5159.

Ibáñez, M.A., M.M. Cavanagh, N.C. Bonamico, y M.A. Di-Renzo. 2006. Análisis gráfico mediante Biplot del comportamiento de híbridos de maíz. RIA 35(3):83-93. 
Ibáñez, M.A., M.A. Di-Renzo, S.S. Samame, N.C. Bonamico, and M.M. Poverene. 2001. Genotype-environment interaction of lovegrass forage yield in the semi-arid region of Argentina. Arg. J. Agric. Sci. 137:329-336. doi:10.1017/ S0021859601001423

Legarda-López, D., G. Benavides-Cuesta, y H. Ruiz-Erazo. 2015. Respuesta del pasto raigrás Aubade (Lolium sp.) a dosis de silicio en interacción con diferentes dosis de NPK. Biotecnol. Sector Agropecu. Agroind. 13(1):99-109.

López, J. 2009. Estudio de los recursos fitogenéticos del complejo Festuca - Lolium. Tesis Ph.D., Universidad de Santiago de Compostela, Santiago de Compostela, ESP.

Lozano-del-Río, A., V. Zamora-Villa, L. Ibarra-Jiménez, S. Rodríguez-Herrera, E. de-la-Cruz-Lázaro, y M. de-la-Rosa-Ibarra. 2009. Análisis de la interacción genotipo-ambiente mediante el modelo AMMI y potencial de producción de triticales forrajeros (X Triticosecale Wittm). Univ. Cienc. 25(31):81-92.

Lozano-Ramírez, A., A. Santacruz-Varela, F. San-Vicente-García, J. Crossa, J. Burgueño, y J. Molina-Galán. 2015. Modelación de la interacción genotipo $\times$ ambiente en rendimiento de híbridos de maíz blanco en ambientes múltiples. Rev. Fitotec. Mex. 38:337-347.

Mejía, J. 2014. Evaluación de la interacción genotipo por ambiente para variedades transgénicas de algodón Gossypium hirsutum L. Tesis M.Sc., Universidad Nacional de Colombia, Palmira, COL.

Méndez, D.G., K. Frigerio, M. Costa, J. Mattera, N. Romero, L. Fontana, L. Romero, P. Barbera, A. Ré, F. Moreyra, J. Otondo, M. Cicchino, M. Bailleres, G. Melani, J. Esquiaga, J. Lavandera, J.J. Gallego, y F. Neira. 2014. Interacción genotipo × ambiente y su asociación con variables climáticas en cultivares de Lolium multiflorum lam. Argentina. En: D. Méndez, y A. Otero, editores, Memoria Técnica 2013-2014. INTA, Buenos Aires, ARG. p. 113-116.

Namorato, H, G. Vieira, L. Vagno, L. Rodrigues, R. Oliveira, and E. Mantovani. 2009. Comparing biplot multivariate analyses with Eberhart and Russell' method for genotype x environment interaction. Crop Breed. Appl. 9:299-307.

Vallejo, F., y E. Estrada. 2013. Mejoramiento genético de plantas. $2^{\text {da }}$ ed. Universidad Nacional, Palmira, COL.

Vargas, E., J. Vargas, y D. Baena. 2016. Análisis de estabilidad y adaptabilidad de híbridos de maíz de alta calidad proteica en diferentes zonas Agroecológicas de Colombia. Acta Agron. 65:72-79. doi:10.15446/acag.v65n1.43417

Vega, P. 1988. Introducción a la teoría de la genética cuantitativa con especial referencia al mejoramiento de plantas. Universidad Central de Venezuela, Caracas, VEN. 